\title{
Determination of Normal Acceleration in Planar Mechanisms by Means of Instantaneous Centres of Rotation
}

\author{
Stevan R. Đorđević ${ }^{*}$, Radovan R. Bulatović ${ }^{2}$ \\ ${ }^{1}$ Faculty of Mechanical Engineering, Belgrade \\ 2 The Faculty of Mechanical and Civil Engineering in Kraljevo of the University of Kragujevac
}

\begin{abstract}
The paper presents a new method for determination of normal accelerations which is based on the geometry of mechanisms. Every closed four-member kinematic group in planar mechanisms has the following characteristic: the vector of sum of relative normal accelerations between the kinematic pairs along the contour of the group is collinear with the line defined by relative instantaneous centres of rotation of the group members that are not in direct contact. This key characteristic of planar mechanisms allows the possibility of simple vector determination of unknown "normal" components of acceleration in a mechanism on the basis of the known, given or unit normal acceleration of the driving member. When two members of a closed kinematic group constitute a sliding pair, constant relationship between the intensity of the Coriolis (and relative normal) acceleration of the kinematic pair with the relative normal acceleration of that member of the sliding pair whose constant centre of rotation lies on the contour can be established. The relationship is defined by absolute and relative instantaneous centres of the sliding pair. Determination of acceleration does not require that characteristic velocities of the mechanism members should be known.
\end{abstract}

Key words: Instantaneous centres of rotation, Normal acceleration, Coriolis acceleration, Planar mechanism, Sliding pair

\section{INTRODUCTION}

All classical methods for determination of acceleration in mechanisms are vector ones. The lines of vectors of tangential accelerations are defined by the kinematic scheme of the mechanism, and their directions and intensities result from the solutions of vector equations (or relations). The kinematic scheme of mechanisms determines lines and directions of vectors of absolute and relative normal accelerations of characteristic points, while their intensities are calculated. For the calculation of intensities of normal accelerations, it is necessary to know absolute and relative velocities of the corresponding points of the members, i.e. the kinematic pairs in mechanisms.

References [1-5], among others, present the methods of kinematic analysis of mechanisms. In the kinematic analysis, the method of determination of velocities by means of instantaneous centres of rotation by the application of Aronhold-Kenedy theorem is also given.

Penock et. al. [6] presents a graphical technique to locate the centre of curvature of the path traced by a coupler point of a planar, single-degree-of-freedom, geared seven-bar mechanism, Also, Penock et. al. [7] presents a graphical technique to obtain the radius of curvature of the path traced by a coupler point of a planar, single-degree-of-freedom, indeterminate eight-bar linkage commonly rererred to as double flier linkage. In papers $[6,7]$, it is shown that the point coincident with the instantaneous centre of zero velocity for this link cannot be obtained from Aronhold-Kenedy theorem.

$\mathrm{Wu}$ et. al. [8] presents animation program based on the curvature theory for the planar four-bar linkage mechanism. The complete kinematics curvature analyses of the planar four-bar linkage mechanism depend on the variation of input link angle, angular velocity and angular acceleration are performed by this program.

This paper presents a new method for determination of normal accelerations based on the geometry of mechanisms. The method requires knowledge of positions of instantaneous centres of rotation, too. Accelerations are determined on the basis of the known, given or unit acceleration of the point of the driving member, without knowing the velocities of points, i.e. the angular velocities of the mechanism members.

The method is illustrated through two theses, which are also proved in the paper on the examples of planar mechanisms. The first thesis proves that the vector of sum of relative normal accelerations between kinematic pairs along the group contour is collinear with the line defined by relative instantaneous centres of rotation of the group members that are not in direct contact. The second thesis proves that in the sliding pair the constant relationship between the intensity of the Coriolis (and relative normal) acceleration of the kinematic pair and the relative normal acceleration of that member of the sliding pair whose constant centre of rotation lies on the contour can be established.

In paper [9], Đorđević showed, on the example of a four-bar linkage, how all components of tangential acceleration of characteristic points of a mechanism can be directly determined, without knowing the velocities, in the function of the known or unit acceleration of the driving member of the mechanism . Also, in paper [10] Đorđević showed how unknown components of acceleration are determined on the example of a mechanism which is added by a two-member group Assura with two rotating and one sliding constraints. The method is geometric and does not require knowledge of velocities in the mechanism. 
By analysing the other professional literature, the papers determining accelerations in a similar way have not been found.

\section{DETERMINATION OF ACCELERATION BY THE METHOD OF COUPLED CENTRES OF ROTATION}

Let us observe a part of a planar mechanism with a large number of members which contains a closed fourmember kinematic group (Figure 1). The arbitrarily designated members $3,4,5$ and 6 constitute a closed kinematic chain in the observed mechanism. The instantaneous centres of rotation $N(35)$ and $M(46)$ made by those members of the kinematic chain that are not in direct contact are known. Permanent collinearity of the vector of sum of relative normal accelerations along the contour of the chain $\overline{R S}$ :

$$
\overrightarrow{R S}=\vec{a}_{C n}^{H}+\vec{a}_{B n}^{C}+\vec{a}_{H_{3} n}^{B}+\vec{a}_{H_{6} H_{3} c o r},
$$

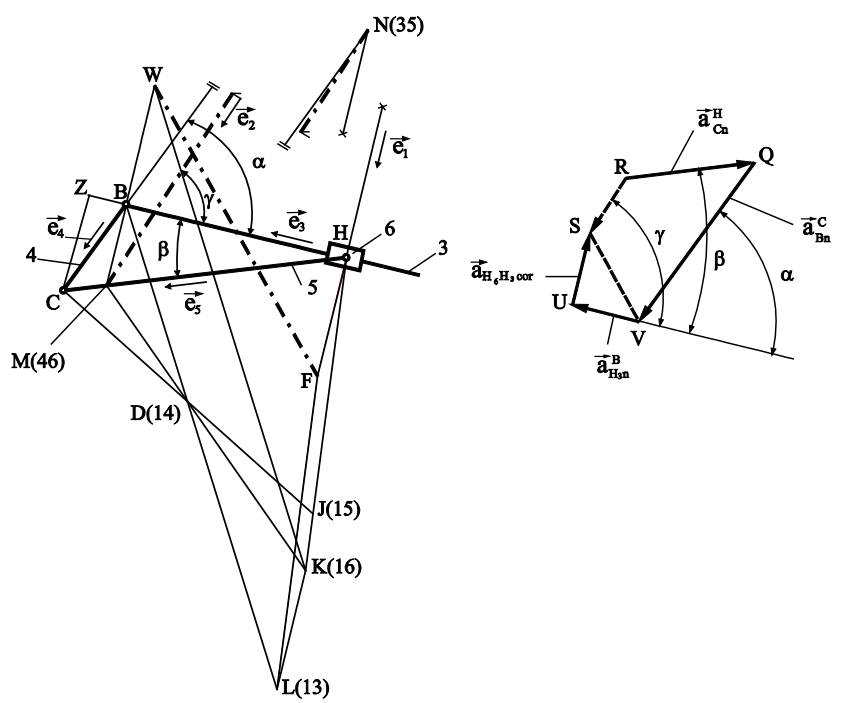

Figure 1: Part of the mechanism with the members 3 and 4, and the additional groupl Assura (5 and 6) form a closed kinematic contour. The resulting vector $R S$ is colinear with $M N$.

and with the direction of coupled instantaneous centres $\overline{N M}$, enables determination of unknown components of acceleration.

The members 3 and 6 form a sliding kinematic couple with a straight guide. The constant centre of rotation $\mathrm{B}$ of the member 3 lies on the closed contour. If the relative normal acceleration of the point $\mathrm{H}$ around the point $\mathrm{B}$ of the member $3-\vec{a}_{H_{3} n}$ (Figure 1), then the resulting vector $\vec{a}_{H_{3} n}+\vec{a}_{H_{6} H_{3} \text { cor }}$ is collinear with the line FW, which can be used during determination of unknown accelerations in the contour. Definition of the line FW is presented in the second part of the paper in a detailed way.

Interpretation of the method will be performed on the mechanism whose kinematic scheme is presented in Figure 3. The instantaneous centres of rotation $S(13)$ and $Q(24)$ are determined. The mechanism realizes its drive through the member 2 .

\subsection{The first thesis}

Let $\overline{P M}$ be the intensity of the currently unknown normal acceleration of the point $A_{4}$ of the guide 4 (Figure 2)

$$
\overline{P M}=\left|\vec{a}_{A_{4} n}\right|=\overline{A C} \cdot \omega_{4}^{2} .
$$

Determination of the intensity of the Coriolis acceleration between the coincident points $\mathrm{A}\left(A_{3}\right.$ and $\left.A_{4}\right)$ of the members 3 and $4, \vec{a}_{A_{3} A_{4} c o r}$ which corresponds to the intensity $\overline{P M}$ is performed according to the following procedure:

The centre of rotation of the sliding pair member which lies on the closed contour (in this case, $\mathrm{C}$ of the member 4) and the absolute instantaneous centre of the second member of the pair $S(13)$ form, with the sliding pair, the rhomboid CSAW whose diagonal SW defines the relationship between the intensities of the mentioned accelerations. The line PN, which is parallel to SW (Figure 2), and $\overline{P M}$ determine the intensity $\overline{M N}$ :

$$
\overline{M N}=\left|\vec{a}_{A_{3} A_{4} c o r}\right|,
$$

which corresponds to the unknown intensity $\overline{P M}$.
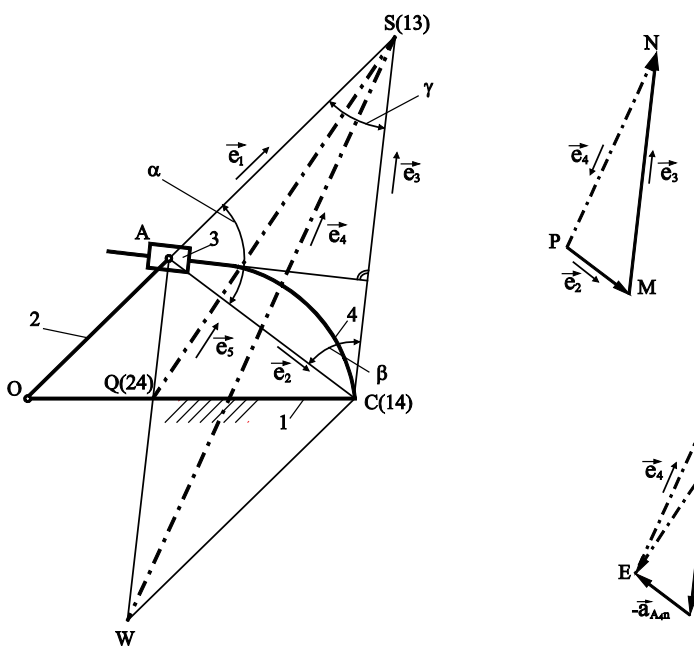

Figure 2: The instantaneous centres $S$ and $Q$ with the line $W S$ define the intensities of all relative normal accelerations in the Geneva mechanism on the basis of the known or unit normal acceleration of the memeber 2.

\subsection{The second thesis}

It is known that normal acceleration of the point $\mathrm{A}$ of the driving member 2 (Figure 2), whose intensity is defined by the expression:

$$
\overline{F G}=\left|\vec{a}_{A_{2} n}\right|=\overline{O A} \cdot \omega_{2}^{2} .
$$

The straight line through $\mathrm{F}$, parallel with $\overline{Q S}$, and the straight line through $\mathrm{G}$, parallel with $\overline{W S}$, define the point E, i.e. the intensities $\overline{P M}$ and $\overline{M N}$.

\subsection{Proof of the first thesis}

a) In case of a kinematic pair with the straight guide (Figure 3)... 
The line defined by the relative centre of rotation of the sliding pair member which lies on the closed contour (in this case the point $B(34)$ of the member 3 ) and the absolute instantaneous centre of the same member $N(13)$, drawn through the absolute instantaneous centre of the other member of the sliding pair ( $M(16)$ of the member 6) intersects the straight line formed by the mentioned centre $B(34)$ and the coupling centre of the sliding pair $G(36)$ at the point Q. In the same way, the line is defined by the relative centre of rotation of the other member of the sliding pair $F(65)$ and its absolute centre of rotation $M(16)$, drawn through $N(13)$, intersects the straight line formed by the belonging instantaneous centres $F(65)$ and $G(36)$ at the point W.

The line $Q W$ defines the relationship between the intensities of acceleration $\vec{a}_{F_{6} F_{3} \text { cor }}$ and $\vec{a}_{F_{3} n}$ (Figure 3). If $\overline{V S}$ is the intensity of relative normal acceleration between the points $\mathrm{F}$ and $\mathrm{B}$ of the member 3 ,

$$
\overline{V S}=\left|\vec{a}_{F_{3} n}\right|=\overline{F B} \cdot \omega_{3}^{2},
$$
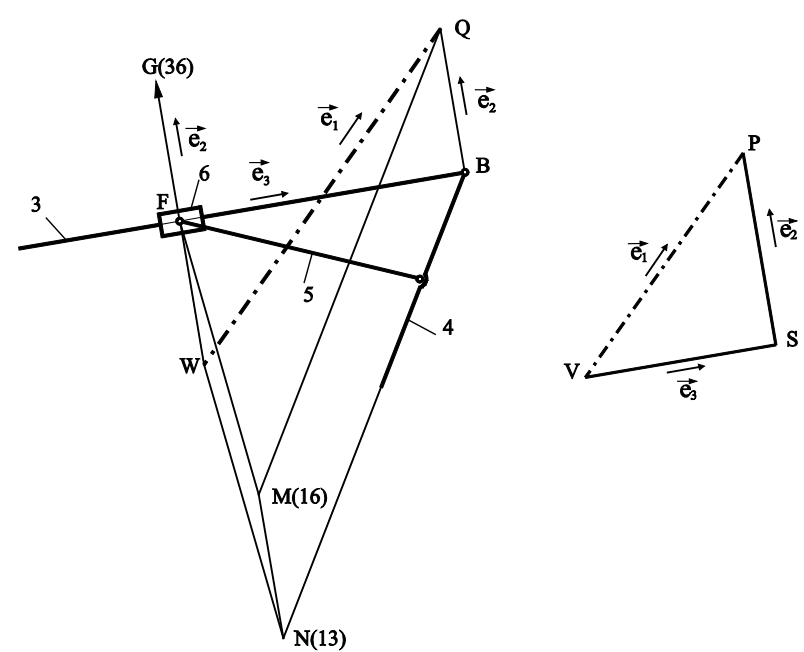

Figure 3: The line $W Q$ defines the relation between the intensities of: relative normal acceleration between the points $F$ and $B$ of the guide 3 and the Coriolis acceleration in the sliding pair 3-6

then the intensity of the Coriolis acceleration $\overline{P S}$ between the members of the sliding pair 3-6 is determined by the expression:

$$
\overline{P S}=\left|\vec{a}_{F_{6} F_{3} c o r}\right|=2 \omega_{3} \cdot\left|\vec{v}_{F_{6} F_{3}}\right| .
$$

The vector defining the line $\overline{W Q}$ is determined by the expression:

$$
\overline{W Q} \cdot \vec{e}_{1}=\overline{W F} \cdot \vec{e}_{2}+\overline{F B} \cdot \vec{e}_{3}+\overline{B Q} \cdot \vec{e}_{2} .
$$

Since $\overline{W F}=\overline{N M}$, i.e. $\overline{Q B}=\overline{N M}$ (from the parallelogram WFMN, i.e. NMQB), the expression (7) by replacement obtains the form:

$$
\overline{W Q} \cdot \vec{e}_{1}=\overline{F B} \cdot \vec{e}_{3}+2 \overline{N M} \cdot \vec{e}_{2} .
$$

From the conditions of collinearity, this relation follows:

$$
\left(\overline{V S} \cdot \vec{e}_{3}+\overline{S P} \cdot \vec{e}_{2}\right) \times\left(\overline{F B} \cdot \vec{e}_{3}+2 \overline{N M} \cdot \vec{e}_{2}\right)=0
$$

After vector multiplication, the expression (9) obtains the form:

$$
\overline{S P}=\frac{2 \overline{V S} \cdot \overline{N M}}{\overline{F B}} \text {, i.e. after the replacement of } \overline{V S}
$$

with the expression (5)

$$
\begin{aligned}
& \overline{S P}=\frac{2 \overline{F B} \cdot \omega_{3}^{2} \cdot \overline{N M}}{\overline{F B}}=2 \omega_{3} \cdot\left(\omega_{3} \cdot \overline{N M}\right)= \\
& =2 \omega_{3} \cdot\left|\vec{v}_{F_{6} F_{3}}\right|=\mid \vec{a}_{F_{6} F_{3}} \text { cor } \mid,
\end{aligned}
$$

is obtained, which proves the statement that the line WQ defines the relationship between the intensities of the mentioned accelerations.

b) In case of a kinematic pair with the arc guide (Figure 4)..
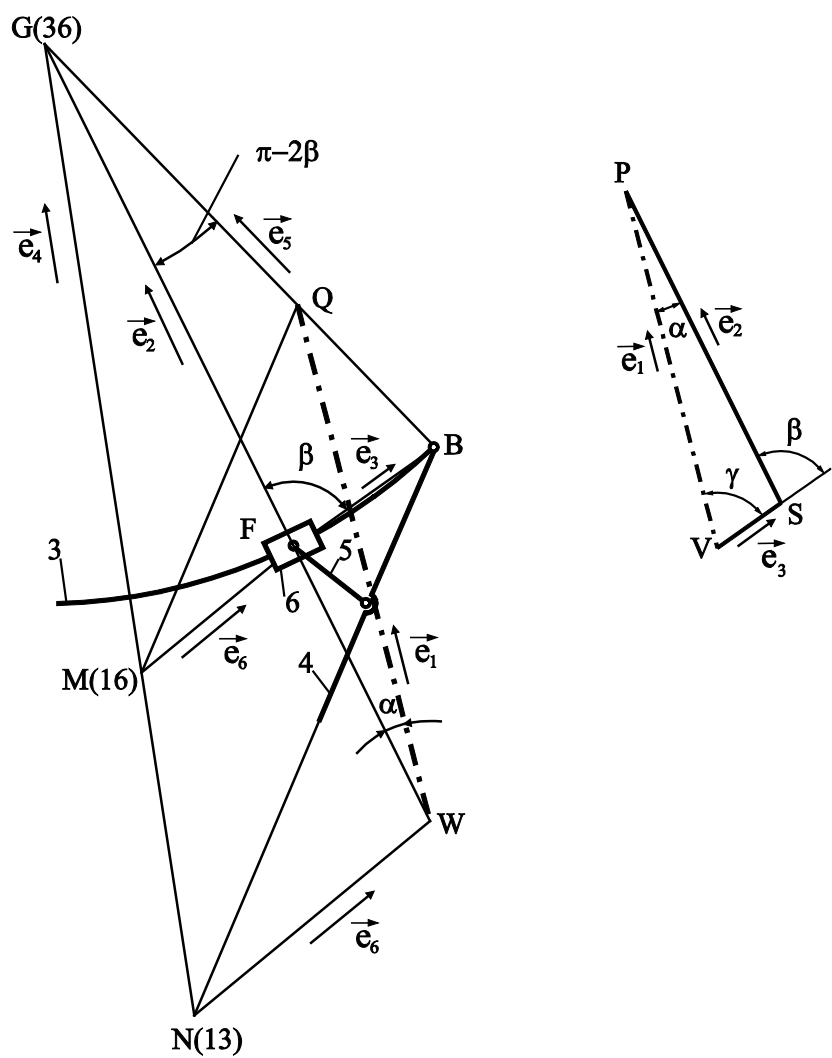

Figure 4: The line $W Q$ defines the relation between the intensities of: relative normal acceleration between the points $F$ and $B$ of the guide 3 and the Coriolis acceleration in the sliding pair 3-6

The line defined by the relative centre of rotation of one member of the sliding pair which lies on the closed contour (in this case the point $B(34)$ of the member 3 ) and the absolute instantaneous centre of the same member $N(13)$, drawn through the absolute instantaneous centre of the other member of the sliding pair $(M(16)$ of the member 6), intersects the straight line formed by the mentioned $B(34)$ and the coupling centre of the sliding pair $G(36)$ at the point Q. In the same way, the line defined by the relative centre of the other member of the 
sliding pair on the contour $F(65)$ and its absolute instantaneous centre $M(16)$, drawn through the absolute instantaneous centre of the first member of the pair $N(13)$, intersects the straight line formed by the mentioned centre $F(65)$ and the coupling centre of the sliding pair $G(36)$ at the point $\mathrm{W}$.

The line QW has the key role in defining the relationship between the intensities of relative normal accelerations between the F-B of the guide and the sum of the Coriolis and relative normal accelerations between the slider and the guide (Figure 4). If $\overline{V S}$ is the intensity of relative normal acceleration between the points $\mathrm{F}$ and $\mathrm{B}$ of the member $3 \overline{V S}=\left|\vec{a}_{F_{3} n}\right|=\overline{F B} \cdot \omega_{3}^{2}$, then $\overline{S P}$ is the sum of intensities of the Coriolis and relative normal accelerations between the members of the sliding pair.

The relation:

$$
\overline{V S} \cdot \sin \gamma=\overline{P S} \cdot \sin \alpha,
$$

follows from the triangle $\triangle P V S$ (Figure 4), i.e. after arranging, the following is obtained:

$$
\overline{P S}=\left(\frac{\sin \beta \cdot \cos \alpha}{\sin \alpha}-\cos \beta\right) \cdot \overline{V S} .
$$

The relation:

$$
\overline{G W} \cdot \sin \alpha=\overline{G Q} \cdot \sin (\pi-2 \beta+\alpha),
$$

follows from the triangle $\triangle G W Q$ and, after rearranging, it obtains the form:

i.e.,

$$
\frac{\overline{G W}}{\overline{G Q}}=\frac{\sin (2 \beta) \cdot \cos \alpha}{\sin \alpha}-\cos (2 \beta),
$$

$$
\frac{\cos \alpha}{\sin \beta}=\frac{1}{\sin (2 \beta)}\left[\frac{\overline{G W}}{\overline{G Q}}+\cos (2 \beta)\right] .
$$

By replacing the expresion (14) in (12), the following is obtained:

$$
\overline{P S}=\left\{\frac{\sin \beta}{\sin (2 \beta)}\left[\frac{\overline{G W}}{\overline{G Q}}+\cos (2 \beta)\right]-\cos \beta\right\} \cdot \overline{V S} .
$$

By using the similarities of the triangles in Figure 4 and the equation $\overline{G F}=\overline{G B}$, the following relation can be derived:

$\frac{\overline{G W}}{\overline{G Q}}=\left(\frac{\overline{G N}}{\overline{G M}}\right)^{2}$, and by replacing it in (15) and by arranging, the following is obtained:

$$
\overline{P S}=\frac{1}{2 \cos \beta}\left[\left(\frac{\overline{G N}}{G M}\right)^{2}-1\right] \cdot \overline{V S} \text {. }
$$

As $\cos \beta=\frac{\overline{F B}}{2 \overline{G F}}$ and $\overline{V S}=\left|\vec{a}_{F n}^{B}\right|=\overline{F B} \cdot \omega_{3}^{2}$, there follows:

$$
\overline{P S}=\overline{F G}\left[\left(\frac{\overline{G N}}{G M}\right)^{2}-1\right] \cdot \omega_{3}^{2} .
$$

Taking into account that $\overline{M G} \cdot \omega_{6}=\overline{N G} \cdot \omega_{3}^{2}$, it can be written that:

$$
\begin{aligned}
& \overline{P S}=\overline{F G} \cdot\left(\omega_{6}^{2}-\omega_{3}^{2}\right)= \\
& =\overline{F G} \cdot\left(\omega_{6}^{2}-2 \omega_{6} \omega_{3}+\omega_{3}^{2}+2 \omega_{6} \omega_{3}-2 \omega_{3}^{2}\right) \\
& \overline{P S}=\overline{F G} \cdot\left[\left(\omega_{6}-\omega_{3}\right)^{2}+2 \omega_{3} \cdot\left(\omega_{6}-\omega_{3}\right)\right]= \\
& =\frac{\left[\left(\omega_{6}-\omega_{3}\right) \cdot \overline{F G}\right]^{2}}{\overline{F G}}+2 \overline{F G} \cdot\left(\omega_{6}-\omega_{3}\right) \cdot \omega_{3} \\
& \overline{P S}=\frac{\left|\vec{v}_{F_{6} F_{3} r e l}\right|^{2}}{\overline{F G}}+2\left|\vec{v}_{F_{6} F_{3} r e l}\right| \cdot \omega_{3}=. \\
& =\left|\vec{a}_{F_{6} F_{3} n}\right|+\left|\vec{a}_{F_{6} F_{3} c o r}\right|
\end{aligned}
$$

which proves this thesis.

c) In the case of the Geneva mechanism (Figure 2)...

The proof of this equation is presented by the following procedure. Let us orientate the line segments $\overline{A S}, \overline{A C}, \overline{C S}, \overline{W S}$ and $\overline{Q S}$ by the unit vectors $\vec{e}_{1}, \vec{e}_{2}, \vec{e}_{3}, \vec{e}_{4}$ and $\vec{e}_{5}$, respectively. The expressions for the vectors:

$$
\begin{aligned}
& \overline{W S} \cdot \vec{e}_{4}=\overline{W A} \cdot \vec{e}_{3}+\overline{A C} \cdot \vec{e}_{2}+\overline{C S} \cdot \vec{e}_{3}=, \\
& =\overline{A C} \cdot \vec{e}_{2}+2 \overline{C S} \cdot \vec{e}_{3} \\
& \overrightarrow{P N}=\vec{a}_{A_{4} n}+\overrightarrow{M N} .
\end{aligned}
$$

can be written.

From the conditions of collinearity of the vectors (21) and (22), there follows the similarity:

$$
\frac{2 \overline{C S}}{\overline{M N}}=\frac{\overline{A C}}{\overline{P M}} \Rightarrow \overline{M N}=\frac{2 \overline{C S} \cdot \overline{P M}}{\overline{A C}},
$$

so that by replacing (2) in (23) and by further arranging, the following is obtained:

$$
\begin{aligned}
& \overline{M N}=2 \overline{C S} \cdot \omega_{4}^{2}=2\left(\overline{C S} \cdot \omega_{4}\right) \cdot \omega_{4}= \\
& =2 v_{A_{3} A_{4}} \cdot \omega_{4}=\left|\vec{a}_{A_{3} A_{4} c o r}\right|,
\end{aligned}
$$

which proves the equation (3), i.e. it is shown that this acceleration is the Coriolis one and that the line $\overline{W S}$ defines the relationship between these two accelerations.

\subsection{Proof of the second thesis}

In the case of a four-member group in which all members are moving (Figure 1)...

The sum of relative normal accelerations along the closed contour $\mathrm{CH}_{6} \mathrm{H}_{3} \mathrm{BC}$ can be defined by the vector:

$$
\overrightarrow{R S}=-\overline{R Q} \cdot \vec{e}_{5}+\overline{Q V} \cdot \vec{e}_{4}+\overline{V U} \cdot \vec{e}_{3}-\overline{U S} \cdot \vec{e}_{1} .
$$

Simultaneously, the line of the coupled centres of rotation can be defined by the expression:

$$
\overline{N M} \cdot \vec{e}_{2}=\overline{N H} \cdot \vec{e}_{1}+\overline{H M} \cdot \vec{e}_{5}
$$

The proof that these two vectors are collinear is that their vector product has the value of zero:

$$
\overrightarrow{N M} \cdot \vec{e}_{2} \times \overrightarrow{R S}=0 \text {. }
$$

The intensities of vectors of normal accelerations along the contour are:

$$
\overline{R Q}=\left|\vec{a}_{C n}\right|=\overline{C H} \cdot \omega_{5}^{2},
$$




$$
\begin{aligned}
& \overline{Q V}=\left|\vec{a}_{B n}^{C}\right|=\overline{B C} \cdot \omega_{4}^{2}, \\
& \overline{V U}=\left|\vec{a}_{H n}^{B}\right|=\overline{B H} \cdot \omega_{3}^{2}, \\
& \overline{U S}=\left|\vec{a}_{H_{6} H_{3} c o r}\right|=2 \overline{L K} \cdot \omega_{3}^{2} .
\end{aligned}
$$

By multiplying the vector members and their grouping, the expression (27) obtains the form:

$$
\begin{aligned}
& \overline{N M} \cdot \vec{e}_{2} \times \overrightarrow{R S}=\overline{N H} \cdot \overline{C H} \cdot \cos \beta \cdot \omega_{5}^{2}+ \\
& +(\overline{H M} \cdot \overline{C B} \cdot \sin (\alpha-\beta)-\overline{N H} \cdot \overline{C B} \cdot \cos \alpha) \cdot \omega_{4}^{2}- \\
& -(\overline{N H} \cdot \overline{B H}+\overline{H M} \cdot \overline{B H} \cdot \sin \beta+2 \overline{H M} \cdot \overline{K L} \cdot \cos \beta) \cdot \omega_{3}^{2}=0 .
\end{aligned}
$$

From the conditions of the same velocities at the poles $N(35)$ and $B(34)$, there follow the relations:

$\omega_{5}=\left(\frac{\overline{K L}}{\overline{H N}}+1\right) \cdot \omega_{3}$ and $\omega_{4}=\left(\frac{\overline{K L}}{\overline{B M}}+1\right) \cdot \omega_{3}, \quad$ so by replacing in (32), the following is obtained:

$$
\begin{aligned}
& \overline{Z H} \cdot \overline{N H} \cdot\left(\frac{\overline{H F}+\overline{H N}}{\overline{H N}}\right)^{2}+ \\
& +(\overline{B H} \cdot \overline{C Z}-\overline{M B} \cdot \overline{Z B}-\overline{Z B} \cdot \overline{N H}) \cdot\left(\frac{\overline{F H}+\overline{B M}}{\overline{B M}}\right)^{2}- \\
& -\overline{N H} \cdot \overline{B H}-\overline{M B} \cdot \overline{B H}-2 \overline{B H} \cdot \overline{F H}=0 .
\end{aligned}
$$

After squaring and mutual multiplying of parts of the expression, identical products of multiplication with the opposite signs appear and they are immediately reduced. Further simplification of (33) is allowed by the relations:

$$
\frac{\overline{Z C}}{\overline{C H}}=\frac{\overline{M B}}{\overline{B H}} \text { i } \frac{\overline{Z B}}{\overline{Z C}}=\frac{\overline{B H}}{\overline{N H}},
$$

obtained from the similarities: $\triangle C Z H \cong \triangle M B H$ and $\triangle C Z B \cong \triangle N H B$

$$
\begin{gathered}
\frac{\overline{F N}^{2}}{\overline{N H}} \cdot\left(\overline{Z H}-\frac{\overline{Z C} \cdot \overline{B H}}{\overline{M B}}\right)+\frac{\overline{Z H} \cdot \overline{F H}^{2}}{\overline{M B}}- \\
-\frac{\overline{Z C} \cdot \overline{B H} \cdot \overline{F H}^{2}}{\overline{M B}^{2}}-\frac{2 \overline{F H} \cdot \overline{Z C} \cdot \overline{B H}}{\overline{M B}}+2 \overline{F H} \cdot \overline{Z H}=0 .
\end{gathered}
$$

And further, by dividing the expression by $\overline{F H}$ (with the condition $\overline{F H} \neq 0$ ) and by reducing, the following is obtained:

$$
\frac{\overline{Z H} \cdot \overline{F H}}{\overline{M B}}-\frac{\overline{F H} \cdot \overline{Z H}}{\overline{M B}}-2 \overline{Z H}+2 \overline{Z H}=0,
$$

which confirms the thesis that the vectors are collinear.

In the case of the Geneva mechanism (Figure 2)...

The sum or relative acceleration along the contour $\mathrm{OA}_{3} \mathrm{~A}_{4} \mathrm{CO}$ can be defined by the vector:

$$
\overrightarrow{F E}=\vec{a}_{A n}-\vec{a}_{A_{3} A_{4} c o r}-\vec{a}_{A_{4} n} \text {. }
$$

Simultaneously, the coupled line through the instantaneous centres of rotation $Q(24)$ and $S(13)$ is also defined by the vector

$$
\overline{Q S} \cdot \vec{e}_{5}=\overline{Q A} \cdot \vec{e}_{3}+\overline{A S} \cdot \vec{e}_{1}
$$

For proving the collinearity of these two vectors, it is necessary that the values of their vector product should be:

$$
\overrightarrow{F E} \times\left(\overrightarrow{Q S} \cdot \vec{e}_{5}\right)=0 \text {. }
$$

Let us express the resulting vector (36) through the parameters of the mechanism:

$$
\overrightarrow{F E}=-\overline{O A} \cdot \omega_{2}^{2} \cdot \vec{e}_{1}-2 \omega_{4}\left|\vec{v}_{A_{3} A_{4}}\right| \cdot \overrightarrow{e_{3}}-\overline{A C} \cdot \omega_{4}^{2} \cdot \vec{e}_{2}
$$

As the relation

$$
\left|\vec{v}_{A}\right|=\overline{O A} \cdot \omega_{2}=\overline{A S} \cdot \omega_{4},
$$

can be established through the intensity of the velocity of the point A, then by replacing in (39) and by arranging, the following is obtained:

$$
\overrightarrow{F E}=-\omega_{2}^{2} \cdot\left[\overline{O A} \cdot \vec{e}_{1}+2\left(\frac{\overline{O A}}{\overline{A S}}\right)^{2} \cdot \overline{C S} \cdot \vec{e}_{3}+\left(\frac{\overline{O A}}{\overline{A S}}\right)^{2} \cdot \overline{A C} \cdot \vec{e}_{2}\right]
$$

By replacing (37) and (41) in (38) and by rearranging, it is obtained that:

$$
\begin{aligned}
& \overrightarrow{F E} \times \overrightarrow{Q S} \cdot \vec{e}_{5}= \\
& =-\omega_{2}^{2} \cdot\left[\begin{array}{l}
\overline{O A} \cdot \overline{Q A} \cdot \sin \gamma+\left(\frac{\overline{O A}}{\overline{A S}}\right)^{2} \cdot \overline{A C} \cdot \overline{Q A} \cdot \sin \beta+ \\
+2\left(\frac{\overline{O A}}{\overline{A S}}\right)^{2} \cdot \overline{C S} \cdot \overline{A S} \cdot \sin \gamma+\left(\frac{\overline{O A}}{\overline{A S}}\right)^{2} \cdot \overline{A C} \cdot \overline{A S} \cdot \sin \alpha
\end{array}\right]
\end{aligned}
$$

When the part of the expression in brackets is divided by $\sin \gamma$ and the sine theorem is applied, it can be written in the form:

$$
\begin{aligned}
& \overline{O A} \cdot \overline{Q A}+\frac{\overline{O A}^{2} \cdot \overline{Q A}}{\overline{A S}}-\frac{\overline{O A}^{2} \cdot \overline{C S}}{\overline{A S}}= \\
= & \overline{O A} \cdot \overline{Q A} \cdot\left(1+\frac{\overline{O A}}{\overline{A S}} \cdot\left(1-\frac{\overline{C S}}{\overline{Q A}}\right)\right)=0 .
\end{aligned}
$$

By using the relation $\frac{\overline{C S}}{\overline{Q A}}=\frac{\overline{O S}}{\overline{O A}}$, the expression (43) obtains its final form

$$
1+\frac{\overline{O A}}{\overline{A S}} \cdot\left(\frac{\overline{O A}-\overline{O S}}{\overline{O A}}\right)=0
$$

which proves the collinearity of the vectors $\overrightarrow{F E}$ and $\overrightarrow{Q S}$, i.e. the second thesis is proved.

\section{CONCLUSION}

Determination of unknown normal components of acceleration in a mechanism is simply performed and does nor require knowledge of velocities and angular accelerations of characteristic points and mechanism members. As the procedure is of vector nature, it is characterized by high precision. Relative values of acceleration are directly connected with the geometry of mechanisms, so that the method itself is suitable for graphical application. All graphics packages that are 
nowadays used in computer design and drawing are suitable for the application of this method.

The aim of further research is that this procedure should be simply integrated into the synthesis process when accelerations in that process figure.

\section{REFERENCES}

[1] A.S. Hail, „Kinematics and Linkage design“, PrenticeHall, Inc., 1966.

[2] C. H. Suh, C. W. Radcliffe, ,Kinematics and Mechanisms Design“, John Wiley \& Sons, Inc., New York, 1978.

[3] R. S. Hartenberg, J. Denavit, „Kinematic Synthesis of Linkages", McGraw-Hill Book Company, New York, 1964.

[4] C. H. Chiang, ,Kinematics and design of Planar Mechanisms, Krieger Publishing Company“, Malabar, Florida, 2000.
[5] R. L. Norton, Design of Machinery: An Introduction to the Synthesis and Analysis of Mechanisms and Machines, McGraw-Hill, Third Edition, 2004.

[6] G. R. Pennock, H. Sankaranarayanan, Path curvature of a geared seven-bar mechanism, Mechanism and Machine Theory, 38 (12 ), (2003), 1345-1361.

[7] G. R. Pennock, N. N. Raje, Curvature theory for the double flier eight-bar linkage, Mechanism and Machine Theory, 39 (7), (2004), 665-679.

[8] T. M. Wu, C. K. Chen, „Computer-aided curvature analyses of planar four-bar linkage mechanism“, Applied Mathematics and Computation, 168(2 ) pp.1175-1188, (2005).

[9] S. R. Đorđević, „Determination of the tangential acceleration for a four-bar linkage mechanism using the coupled centers method", Ninth World Congress on the Theory of Machines and Mechanisms, Milano, Vol. 1, 159-161, (1995).

[10] S. R. Đorđević, Determination of accelerations of a characteristic kinematic group by method of coupled centers, FME Transactions, Belgrade, 30(2), pp. 59- 62, (2002). 